the gas, not to employ any other form. I have only now recommended the effervescing draughts as more convenient than the bottled aerated liquids for general employment and private practice, as stated by me in a note at page thirty-one of the work under consideration. This circumstance was more particularly dwelt upon in the first edition of my work, and ought, perhaps, to have been repeated in the present one, but thinking that sufficient evidence had been advanced of the efficacy of carbon and carbonic acid, in their pure forms, the remarks on this particular point were omitted. On the other hand, I did not suppose that, in the present day, any one, excepting Dr. Stevens himself, would suppose the benefit derived in the above instances could be ascribed to the administration of a small quantity of tartrate of soda, or potash; for I inferred that the profession generally had arrived at the same conclusion as myself-viz., that the saline treatment, when trusted to alone, and uncombined with any other remedy, was not only useless, but actually injurious. More than this, $I$ have concluded that the benefit derived, in some instances, when saline remedies were given, was due, not to the employment of these agents, but to the simultaneous administration of carbonic acid gas. This will be evident from the following extract, taken from the introduction to the first edition of my work. While speaking of the variation observed by the employment of the same remedies, when given alone, and when combined with carbonic acid, it was added,-

"As an example, I may mention the different results that have been obtained, with different individuals, by what has been termed the saline treatment. Dr. Stevens, and several other practitioners, affirmed that this treatment was more successful than any other that had been adopted; while, on the contrary, many who had tried the same remedies considered them either useless, or worse than useless. Now, to what are we to ascribe this great discrepancy? Not to the perversion of facts, or a biassed judgment; for, notwithstanding a dispute ensued, which became, to say the least of it, acrimonious and personal, we have no reason to question the honour or the motives of either party, much less of the talented individual most interested in the issue of the question. The solution of the problem I consider to be this:- It appeared, by the report of Sir David Barry, that the patients in the Cold-bath Fields prison were allowed an unlimited use of seltzer-water, to relieve the thirst, and allay the irritability of the stomach; saline effervescing draughts being also added, to check the vomiting when this was severe-a practice followed, to a certain extent, by various other practitioners who adopted the saline treatment. Well, then, might Dr. Stevens say, when asked by Sir David where his cases of collapse were, that his plan of treatment prevented the supervention of that stage of the disease, being well convinced that patients who had an unlimited allowance of soda-water, in the first stages of the disease, would never fall into a state of collapse. But as the exhibition of carbonic acid had no connexion with the theory put forth by Dr. Stevens, and was not even mentioned as an adjuvant, in the first and original communications of this gentleman, others who adopted the same plan of treatment did not add this agent to the saline remedies, and hence the difference in the result."-p. 87.

I should have been glad to append a few remarks in reply to your observations as to the apparent failure of the remedy in cases of complete and confirmed collapse, but, as I fear to trespass further on your indulgence, and as this point has been already dwelt upon in my work, I must refrain; were it not also from a fear of confirming the impression that appears to have been made on the mind of the Reviewerviz., that $I$ am disposed, like most persons who have such a plaything, to ride my hobby too hard, $I$ would have added a few facts confirmative of the interesting communication of your correspondent, contained in the same number, as to the exemption of particular persons, or classes, exposed to an atmosphere of carbonic acid gas, as I have collected a great many examples of the same kind. At present, however, I will conclude by merely expressing a hope that I have not been too prolix, and that these remarks may be deemed worthy a place in the columns of THE LANCET.

I am, Sir, your very obedient servant,

London, August 9th, 1848 .

J. Parkin.

\section{PROFESSIONAL QUACKERY.} To the Editor of THE LANCET.

Sir,-Your continual efforts to benefit our profession, by denouncing quackery, induce me to send the inclosed advertisement, taken from the Liverpool Mail. You will notice that the man claims the honour of being a M.R.C.S. and L.A.C. This is only one among the many instances which constantly come before our eyes of members of our profession degrading their honourable calling by quackish and ungentlemanly practices, induced by the love of filthy lucre; so true are the words of the poet-

$$
\text { "srepe dat indignis." }
$$

How can we be surprised at quackery gaining such a height, when so many cases of it are daily occurring in the profession itself, while the learned bodies of which the culprits are members look on with silent apathy and indifference.

I would ask, through the medium of your valuable publication, whether the council of the College of Surgeons, and other bodies of examiners, conscientiously feel that they fully perform the important duties with which they are entrusted, when they thus wink at the conduct of those who have sworn to observe the laws and regulations, and to uphold the dignity of the college of which they have been admitted members. Undoubtedly, to behave like gentlemen, to treat with proper courtesy their fellow-practitioners, as well as to give the whole profession the benefit of any new mode of treatment, are essential to the dne performance of the oaths taken upon being admitted under the banners of Esculapius.

What a small amount of honourable and gentle feelings, then, must be contained in the breasts of those who blaze forth their own very questionable merits at the expense of truth, and of practitioners infinitely their superiors.

I trouble you, Sir, with these few lines, feeling how necessary it is to have our noble profession cleared of those who are each day contaminating it more and more.-I am, Sir, your obedient servant,

Manchester, July, 1848. M.R.C.S. \& L.A.C.

\section{QUACKERY.}

\section{To the Editor of THE LANCET.}

$\mathrm{SIR}_{3}$-I herewith enclose a piece of paper which was placed in my hands this morning by a dirty boy, on my way to Holborn, through Clerkenwell. Make what use you like of it.

Your obedient servant,

E. B.

"Mr. A. W. Eves, Surgeon and Accoucheur, (member of the Royal College of Surgeons, is in constant attendance for gratuitious consultation at 41, Clerkenwell-green. Patients are visited at their own houses when necessary; and all medicines are prepared from drugs of the purest quality at reasonable prices.-Bleeding, cupping, and tooth-drawing."

\section{To the Editor of Tre Lavoen.}

Str, - I send you a leaf from the advertising sheet of the Dublin Quarterly Journal of Meciical Science, with an advertisement marked. Surely the profession must not complain that newspapers admit quack advertisements when our own journals advertise such humbug as a "medical work." I am, Sir, your obedient servant,

Colchester, August 5, 1848. A Constant Reader.

"Just published, price 1s., free by post, 1s. 6d., the sixth English edition, (translated from the twenty-fourth French edition,

Constipation Desfroyed ; or, exposition of a natural, simple, agreeable, and infallible means of overcoming habitual constipation, and of completely eradicating chronic diseases of the digestive organs, witlout using medicines, or any artificial meaus whatever. (Discovery made in France, by M. Warton.) Confirmed by numerous certificates from eminent physicians, and other persons of the highest respectability, in the United Kingdom, France, and other countries.-Sold by J. Youens, 36, Farringdon-street, London, and all booksellers in the United Kingdom."

\section{AN ECONOMIC FILTER.}

To the Editor of The LanceT.

SIR,-For the last month I have had several applications from medical gentlemen respecting the description of the filter which you favoured me by describing in your valuable journal (last vol., No. 24, p. 637.) They seem to approve of it very much, yet there is a condition which should be attended to-viz., that all the ingredients except the powdered charcoal should be well washed, till the water runs through perfectly clear, and then placed in their respective layers. The river or wreck sand can be obtained at any of the builders, as it is the sand they use.-I remain, yours truly, Bridge-street, Southwark. EDWARD HALL, M.R.C.S.L. 